Fecha de recepción: abril 2021

Fecha de aprobación: mayo 2021

Fecha publicación: junio 2021

\section{El proyecto contemporáneo, entre la tradición y la vanguardia. Reflexiones desde la arquitectura}

Florencio Compte Guerrero ${ }^{(1)}$

\begin{abstract}
Resumen: En la Bienal Panamericana de Arquitectura de Quito de 2014 el jurado entregó el Primer Premio a una pequeña casa construida en caña guadúa en un área rural de la Provincia de Manabí. Dos años más tarde fue premiado el Cabañón DLPM, de ladrillo y bambú, integrado a un acantilado frente al mar, que reconfigura el concepto de espacio al desarrollar una rampa que se convierte en un lugar lúdico para habitar. A éstos, hay que sumar el trabajo de los colectivos Natura Futura, Frontera Sur y Al Borde que están planteando la necesidad de conciliar la vanguardia y la recuperación de la tradición, tanto espacial como constructiva. Algo está cambiando en la arquitectura del mundo y ese cambio se está dando desde el sur de nuestro continente.
\end{abstract}

Palabras clave: tradición - vanguardia - caña guadúa - espacio arquitectónico - arquitectura contemporánea.

[Resúmenes en inglés y portugués en las páginas 101-102]

(1) Doctor en Diseño (Universidad de Palermo) y Arquitecto (Universidad Católica de Santiago de Guayaquil). Profesor Titular Principal de Historia de la Arquitectura e investigador en la Facultad de Arquitectura y Diseño de la Universidad Católica de Santiago de Guayaquil. Es autor de los libros Como el ave fénix. Mirada crítica a la historia de la arquitectura y la ciudad de Guayaquil (2018), Arquitectura Moderna de Guayaquil. 1930-1948 (2018), Arquitectos de Guayaquil (2007) y coautor de Italianos en la arquitectura de Ecuador (2012), Ciudad y Arquitectura República de Ecuador 1850-1950 (2009), Guayaquil al vaivén de la ría (2003), Testimonio y memoria de la arquitectura histórica de Guayaquil (1996), Guayaquil. Lectura histórica de la ciudad (1993), Patrimonio arquitectónico y urbano de Guayaquil (1989), además de artículos y ponencias sobre la historia de la arquitectura de la ciudad de Guayaquil. Ha sido Profesor de la Maestría en Pensamiento Estratégico y Prospectiva para la Educación Superior en la Universidad Naval Rafael Morán Valverde, de la Especialización en Historia en la Universidad Andina Simón Bolívar y de la Maestría en Diseño Arquitectónico en la Universidad Técnica de Ambato, además forma parte del cuerpo académico de la Maestría en Crítica y Diseño Arquitectónico Avanzado de la Universidad Católica de Santiago de Guayaquil. 


\section{Introducción}

En el año 2014 el jurado ${ }^{1}$ de la categoría de Diseño Arquitectónico de la XIX Bienal Panamericana de Arquitectura de Quito de 2014, presidido por el finlandés Juhani Pallasmaa, decidió entregar el Primer Premio Nacional e Internacional a una pequeña casa construida en caña guadúa diseñada por el arquitecto guayaquileño Enrique Mora, en un área rural aislada del recinto Convento de la Provincia de Manabí en Ecuador.

En una especie de manifiesto que precedía al veredicto final, el jurado reflexionaba sobre el porqué de haber elegido esta sencilla obra entre las casi trescientas que se presentaron al concurso:

Aparentemente alrededor del mundo la ideología consumista prevaleciente tiende a elevar a la arquitectura fuera de los niveles fundamentales del habitar y de la experiencia humana; creemos que es necesario ir hacia propuestas de espacios con sentido colectivo en lugar de imágenes comercializadas y de dudoso valor estético.

El jurado considera que la verdadera emoción de la arquitectura puede solo surgir de la realidad de la vida, del reconocimiento de las bases de la existencia y del profundo sentido de construir.

Es sintomático en la situación actual de la arquitectura, que los proyectos más emocionantes frecuentemente surgen bajo condiciones de severas limitaciones, pobreza y un sencillo conocimiento tecnológico. Por tanto, los arquitectos se encuentran obligados a potenciar una nueva visión con el uso de recursos, materiales y técnicas.

Las limitaciones socioeconómicas pueden orientar a los arquitectos a manifestaciones arquitectónicas primarias de relevancia cultural y formal (Pallasmaa, Mozó, Rocha, Compte y Moscoso, 2015, pp. 19-20).

Dos años más tarde, durante la XX Bienal Panamericana de Arquitectura de Quito ${ }^{2}$, en la misma categoría de Diseño Arquitectónico, los premios nacional e internacional fueron para el Cabañón DLPM, ubicado en Las Tunas, en la ecuatoriana Provincia de Manabí, de los arquitectos Alejandro González, Juan Carlos Bamba e Ignacio de Teresa y el premio nacional también para El Tallercito, ubicado en Babahoyo, Provincia de Los Ríos, diseñado por el colectivo Natura Futura.

A éstos, hay que sumar el trabajo de las oficinas de arquitectura Frontera Sur -quien en una propuesta colectiva con Natura Futura fue premiado en la XXI Bienal Panamericana de Arquitectura de Quito-y Al Borde, y de arquitectos como Robinson Vega, quienes están planteando la necesidad de conciliar la vanguardia con la recuperación de la tradición, tanto espacial como constructiva. En ese sentido, se empieza e entender a la contemporaneidad en el diálogo permanente entre lo aprendido a lo largo de los siglos y las nuevas cuestiones proyectuales. 


\section{Lo vernacular y lo contemporáneo}

En general, cuando hablamos de arquitectura vernácula lo hacemos como distinción de la arquitectura académica. Sin embargo, el concepto de vernáculo, va asociado principalmente a la adecuación de la arquitectura al medio, con soluciones eficientes frente a condiciones climáticas determinadas y a la necesidad de haber encontrado soluciones prácticas mediante la constante prueba-error en el uso de materiales y sistemas constructivos existentes en el medio y la transmisión de esos saberes y resultados a las generaciones venideras.

En 1964, Bernard Rudofsky realizó en el Museo de Arte Moderno de Nueva York la exposición Arquitectura sin arquitectos, que presentó una recopilación fotográfica de viviendas tradicionales alrededor del mundo, en las que destacaba la continuidad de los saberes ancestrales en las soluciones climáticas y en el uso de materiales y técnicas constructivas locales como respuesta a las necesidades de habitar.

Más tarde, el propio Rudofsky (2000) se refería a esta arquitectura como aquella que “... no sigue modas, sino que evoluciona en el tiempo casi imperceptiblemente” (p. 13). ¿Pero qué pasa con la arquitectura que, haciendo uso de esa tradición vernacular, ha evolucionado e incorporado una visión contemporánea y de vanguardia?

Para dar respuesta a esta pregunta, resulta útil revisar el planteamiento de Kenneth Frampton (2010, 4a. ed.), cuando al definir al regionalismo crítico, hace una clara referencia a aquella arquitectura que, siendo actual, se enlazaba con la tradición:

El regionalismo crítico suele florecer en estos intersticios culturales que de un modo u otro son capaces de escapar del empuje optimizador de la civilización universal. Su aparición indica que esa noción heredada consistente en un centro cultural dominante rodeado por satélites dependientes y dominados es en última instancia un modelo inadecuado con el que valorar el estado actual de la arquitectura moderna (p. 332).

También Alan Colquhoun desarrolla la idea de cómo podía desarrollarse una arquitectura actual que se basara en la tradición:

El concepto de regionalidad depende de la capacidad para relacionar códigos culturales con regiones geográficas. Está basado en sistemas tradicionales de comunicación en los que el clima, la geografía, las tradiciones artesanales y las religiones son absolutamente determinantes (Colquhoun, 1992, p. 17).

Resulta útil hacer una revisión de cómo se ha conformado la arquitectura vernácula en la costa ecuatoriana. En esa arquitectura converge la transferencia directa de la espacialidad hispana, urbana o rural, junto con la incorporación de materiales y métodos locales -caña guadúa ${ }^{3}$, bijao-quincha ${ }^{4}-\mathrm{y}$ sistemas constructivos precolombinos.

Los grabados que dejaron los viajeros durante la Colonia muestran las casas campesinas con características similares a las contemporáneas, esto es de estructura de caña levantada 
sobre palafitos y cubiertas a dos aguas con techumbre de bijao. De esta manera describen a esas casas los viajeros Jorge Juan y Antonio de Ulloa en el siglo XVIII (1748):

El principal y común de las casas que pueblan las orillas de aquel río desde Guayaquil hacia arriba, se reduce a cañas, de cuyo grueso y particularidades se tocará en su lugar. Con ellas se fabrica todo el techo interior, las paredes, suelo, escaleras de las habitaciones chicas, pasamanos y demás necesario (...) Para formar el techo en las casas grandes sientan la cumbrera principal de madera; $y$ todas las demás vigas, que bajan de ella a descansar en la vertiente son de caña; sobre las cuales entablen con las que se hacen de las mismas; cúbrenlo por defuera con hojas de Vijahua ${ }^{5}$ y queda concluido a poca costa sin mucho trabajo, y con toda la capacidad, y conveniencia, que se apetece.

Esta arquitectura en madera y caña se mantuvo en las áreas urbanas consolidadas de la costa ecuatoriana hasta bien entrado el siglo XX. Su uso se dio no solo por la calidad de los materiales y la experticia de los constructores, porque muchas veces se asentaban en suelos inundables o de baja capacidad portante, sino, también, porque ofrecía mejor resistencia ante los sismos, tal como lo indicaba Carlos Wiener (1960 [1880]):

Los terremotos, que suelen ser bastante prolongados, imprimen a estos edificios oscilaciones que derribarían las de piedra, por sólidas que fueran; en cambio, no producen ningún efecto destructor en esas construcciones que oscilan, crujen y continúan incólumes como en un barco en un mar agitado (p. 451).

También aún se puede encontrar esta arquitectura en sectores periféricos marginales de las ciudades de la región costera ecuatoriana y en los entornos rurales, desarrollada con procesos de autoconstrucción con los mismos materiales y sistemas constructivos de los siglos precedentes y con tipologías espaciales y formales similares.

De esa tradición vernacular surgen contemporáneamente propuestas que valoran su aporte, que rescatan la nobleza de los materiales con la que fue levantada, que ponen en valor los conocimientos ancestrales y que entienden su relación con los modos de vida que la generó.

\section{Del dicho al hecho}

\section{La Casa Convento}

La Casa Convento fue diseñada y construida por el arquitecto Enrique Mora en medio de un bosque de bambú en una zona rural aislada de la Parroquia Convento, perteneciente al Cantón Chone en la Provincia de Manabí, al noroeste del Ecuador. La casa pertenece a la abuela de la esposa del arquitecto, vinculada al trabajo agrícola y a la tradición de la cons- 
trucción vernacular de la región litoral ecuatoriana, por lo que se planteó en la necesidad de establecer un diálogo entre esa tradición y lo contemporáneo.

Mora plantea que el proyecto de la casa es “...una reinterpretación de la arquitectura tradicional vernácula de la costa del Ecuador" (E. Mora, comunicación personal, agosto de 2019), la considera contemporánea “... por el mismo hecho del tiempo en que ha sido construida” y tradicional “... por el uso de elementos con un lenguaje nuevo, aportando, de esa manera, una nueva configuración espacial, a partir de la comprensión y respeto por el lugar, el uso de los recursos materiales, la mano de obra y los conocimientos tradicionales de construcción con los que se contaba" (E. Mora, comunicación personal, agosto de 2019). Destaca la importancia del respeto a la tradición de la autoconstrucción, ya que la familia propietaria de la casa aportó con el ancestral conocimiento del manejo de la caña guadúa -su fecha de corte, el proceso de crecimiento, su tratamiento, etc.- y el conocimiento empírico de construcción de viviendas, mientras que el arquitecto interpretó esos saberes junto con su conocimiento técnico para mejorar el sistema constructivo utilizado. En el veredicto del jurado de la categoría Diseño Arquitectónico de la XIX Bienal Panamericana de Arquitectura de Quito, quien otorgó el primer premio a la obra, tanto nacional como internacional, se indicaba:

El proyecto es una sencilla casa en un área remota del Ecuador, que ha sido construida con recursos modestos utilizando materiales locales, especialmente bambú

(...)

A pesar de sus limitaciones, se desarrolló una arquitectura humana y bella que refleja la elegancia y la inmaterialidad de una arquitectura industrial de alta tecnología, trabajada con medios muy humildes.

(...)

Este proyecto ecuatoriano aporta un ejemplo estimulante a los esfuerzos actuales que se desarrollan alrededor del mundo para diseñar obras arquitectónicas para condiciones limitadas en términos de aceptabilidad, economía, capacidades de construcción y materiales disponibles (Pallasmaa, Mozó, Rocha, Compte y Moscoso, 2015, p. 20).

Mora reflexiona también sobre la necesidad de repensar y replantear la arquitectura contemporánea desde la tradición:

En la actualidad, ante el uso desmedido de los recursos, de la contaminación del planeta y la pérdida de identidad de los pueblos, es importante que la arquitectura aprenda, valore y resignifique la arquitectura tradicional, sin perder la condición de contemporaneidad. Expresando un lenguaje nuevo en el uso de sus formas, con la introducción de tecnologías y nuevos materiales que permitan hacer edificios más eficientes (E. Mora, comunicación personal, agosto de 2019).

La obra ha tenido reconocimientos adicionales, como el premio en diseño arquitectónico de la X Bienal Iberoamericana de Arquitectura y Urbanismo de Sao Paulo del año 2016. 


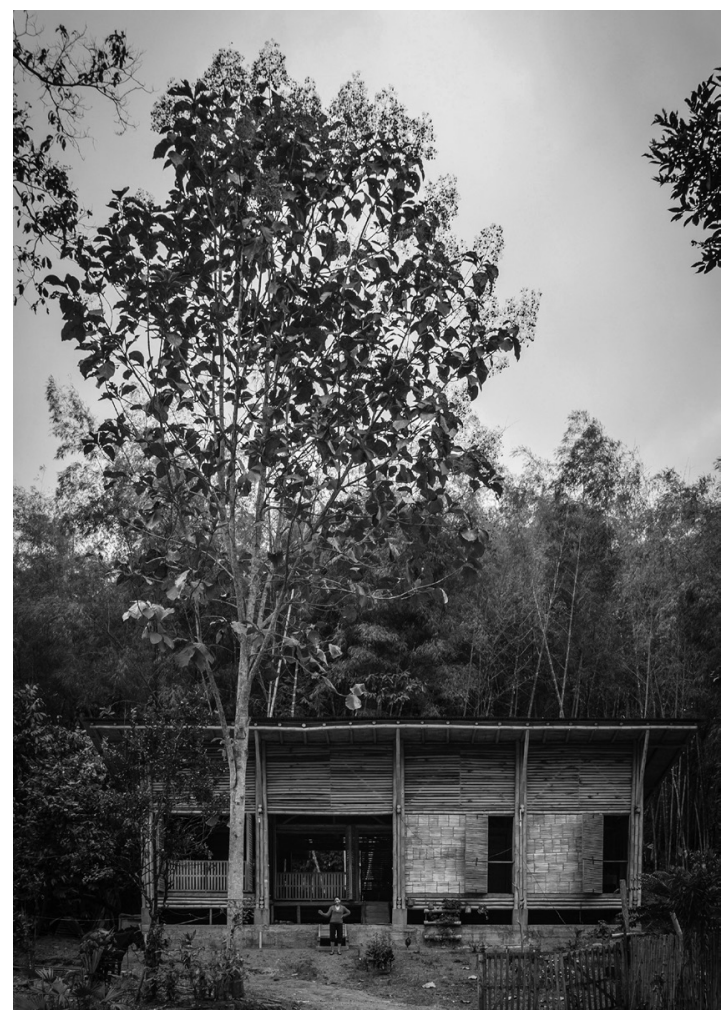

Figura 1. Casa

Convento [Fotografía de Enrique Mora] (Recinto Convento, Chone, Provincia de Manabí, Ecuador). Archivo Enrique Mora Alvarado. Guayaquil, Ecuador.

\section{El Cabañón DLPM}

El pedido de la pareja dueña del terreno era claro: querían una cabaña económica que les permitiera pasar la noche y hacer un seguimiento del terreno cultivable que tenían alrededor. La localización: un terreno en pendiente en una montaña del sector de Ayampe, en la Provincia de Manabí, frente al Océano Pacífico, conocida por su mano de obra experta en construcciones de caña guadúa.

Los mismos dueños presentaron a los arquitectos un dibujo en el que se situaban en la cubierta de la casa -emplazada en la parte más inclinada del terreno- a la que habían accedido por una rampa ubicada en medio de los árboles.

De esta manera, los diseñadores del proyecto: Juan Carlos Bamba, Ignacio de Teresa y Alejandro González, plantearon recuperar el plano inclinado como espacio habitable, capaz de dar acceso a las superficies horizontales que soportan el programa convencional de una casa (sala, comedor, cocina, baño, cama y oficina), que se alinea a lo largo de tres planos inclinados que prolongan la pendiente del terreno y que generan varias cubiertas superpuestas. 
La casa se conforma por una única habitación que da lugar a un espacio continuo, fluido y genérico, articulado por la rampa que funciona como una "extraordinaria costura espacial” (según palabra de los autores) y nexo de unión, por lo que los habituales ritos de paso entre habitaciones, gestionados tradicionalmente por las puertas, son sustituidos por sutiles matices en la forma del suelo.

De esta manera, se plantearon recuperar las tipologías espaciales de casi todas las viviendas de la zona, conformadas por una sola habitación donde la familia está unida en un solo espacio común y democrático, separada solo por el mobiliario.

Constructivamente, una estructura de caña guadúa envuelve el núcleo central de ladrillo a modo de andamiaje. El desarrollo en rampa de la estructura permite su triangulación y el uso de losas de hormigón aligeradas con cascarilla de arroz reduce su peso y el costo total de la construcción.

Alejandro González, uno de los arquitectos del proyecto, reflexiona sobre la contemporaneidad del proyecto y su vinculación con la tradición local:

Si bien la contemporaneidad es una condición temporal determinada por un presente, o momento relativo al tiempo en el que se vive, ésta se encuentra en permanente transformación y, por lo tanto, su arquitectura debe responder a los cambios de necesidad que experimenta. Necesidades en los hábitos de la sociedad que la habita -para qué-, en el uso sostenible de recursos materiales, técnicos y humanos que la construyen -cómo-, así como en el respeto, adaptación y potencialización del lugar en el que se implanta -dónde-.

Tradición deriva del latín tradere (entregar, inspirar) y significa llevar más allá, es un repetir y perfeccionar. La arquitectura tradicional trabaja por repetición. Con cada repetición tipológica que, ya explorada en el pasado, no solo reinterpreta, actualiza y perfecciona un detalle constructivo o una situación espacial, sino que contribuye a reforzar, de una manera natural, la identidad del lugar donde se trabaja. Referentes como la habitación única de la Petit Cabanon de Le Corbusier (1953) o el habitar del plano oblicuo de Claude Parent y Paul Virilio (1963), forman parte de nuestro universo de estudio.

Los proyectos de arquitectura avanzan unos sobre otros. Es una experimentación continua que te obliga a estudiar las cosas y a rehacerlas. Su condición de proyecto-experimento lo convierte en un prototipo, o versión preliminar, de un vehículo a partir del cual se pueden desarrollar otros proyectos. El prototipo posee cierto nivel de imperfección que favorece la posibilidad de mejorar su versión. Los prototipos son productos de investigación, conscientes de sus propias limitaciones, abiertos a compartir sus hallazgos. Todo proyecto debe responder a las necesidades específicas de su existencia y abrir caminos posibles de investigación universal (A. González, comunicación personal, agosto de 2019).

Al preguntarle sobre la contemporaneidad del proyecto y su vinculación con la tradición vernácula del lugar, Ignacio De Teresa contesta: 
Podríamos entender que algo contemporáneo no es algo hecho en respuesta a las cuestiones de su tiempo, sino que el concepto de contemporaneidad es por definición cambiante y siempre atada al presente inmediato, por lo que tiene que ser continuamente revisable. Según esto, la única arquitectura realmente contemporánea es aquella capaz de adaptarse a futuros cambios, de lo contrario dejaría de ser contemporánea inmediatamente después de haber sido construida. Por esta razón, para ser considerado como contemporáneo, el proyecto tiene que hacer referencia a su vez a todo lo anterior, a una revisión continua de lo aprendido en el lugar, que incorpora pequeños momentos de cambio (I. De Teresa, comunicación personal, agosto de 2019).

El Proyecto Cabañón DLPM ${ }^{6}$ ha podido exponerse a una crítica internacional en dos contextos culturales distintos -América Latina y Europa-, y ha obtenido el reconocimiento por parte de sus jurados. Aparte del premio de la Bienal de Arquitectura Panamericana de Quito, en el 2018 el Colegio Oficial de Arquitectos de Granada (España) le concedió el premio García de Paredes en Innovación y Sostenibilidad.

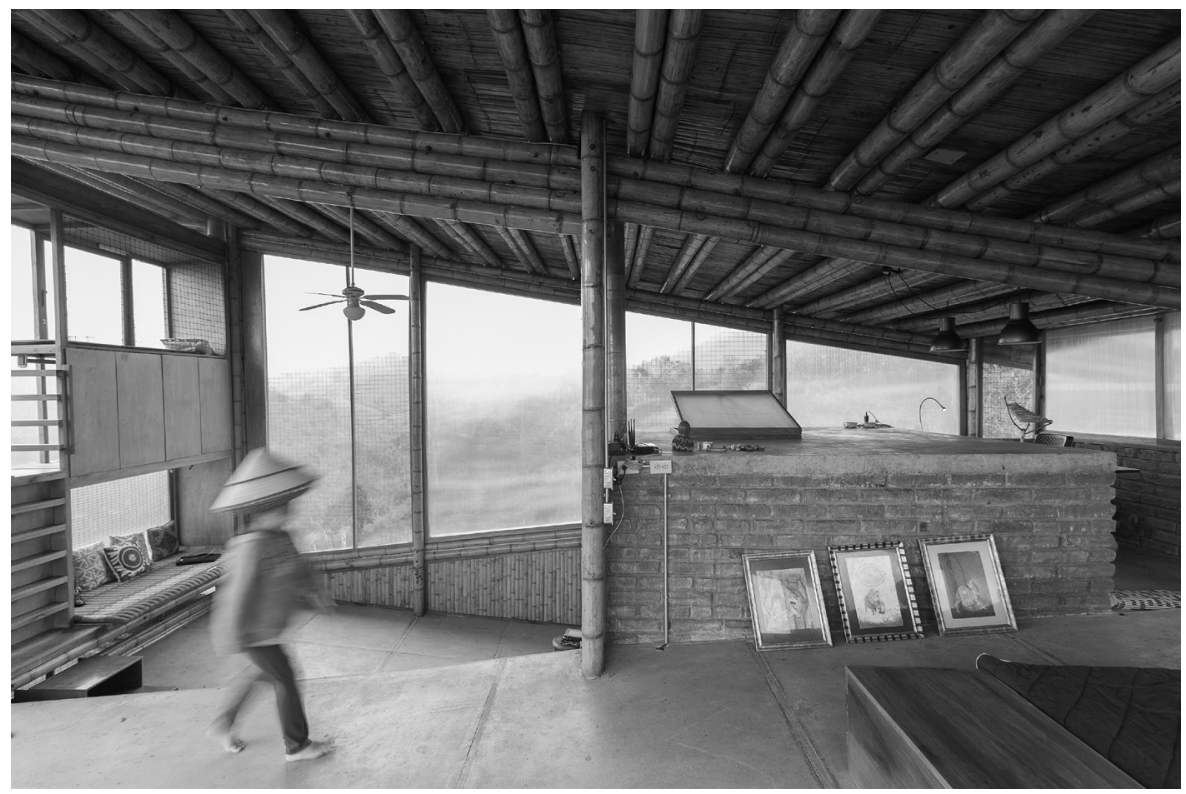

Figura 2. Cabañón DLPM [Fotografía de Juan Carlos Bamba] (Las Tunas, Puerto López, Provincia de Manabí, Ecuador). Archivo arquitectos Bamba, De Teresa y González. Guayaquil, Ecuador. 


\section{La Casa Entre Bloques y La Comuna}

En la Bienal Panamericana de Arquitectura de Quito de 2018 otra obra que aborda lo local y lo tradicional para la generación de una propuesta contemporánea del colectivo Natura Futura obtuvo una Mención Honorífica. Se trata de la Casa Entre Bloques, ubicada en Babahoyo, capital de la Provincia de Los Ríos, en Ecuador

(...) ciudad donde los espacios son sinónimos de estrechez, falta de ventilación, falta de iluminación. Siempre la excusa es la falta de presupuesto. La apuesta es lograr un respiro, una oportunidad de construir con pocos recursos una solución que satisfaga las necesidades reales (José Fernando Gómez, Natura Futura. Arquitectura, 2019).

El equipo de arquitectos enfrentó el desafío de diseñar un refugio para Oscar, “...un comunicador audiovisual, gestor cultural de eventos musicales, espíritu libre y propietario de una parcela de 7x10 m". Como continúan con su descripción: "El proyecto era capturar el espíritu no sólo creativo del poder ocupante, sino que, además, es arrancar esa alma frágil, ligera y evanescente de la pequeña ciudad" (José Fernando Gómez, Natura Futura. Arquitectura, 2019).

El resultado: una vivienda mínima con mampostería de bloques de concreto visto, con grandes ventanales que favorecen la ventilación cruzada y con mobiliario empotrado diseñado también por el mismo colectivo.

$\mathrm{Al}$ reflexionar sobre la arquitectura que desarrollan, los arquitectos de Natura Futura indican:

Nos interesa más el futuro de la arquitectura que la arquitectura del futuro, el redescubrir que el descubrir, el entender y adaptarnos a los procesos, estos que se generan en acciones cotidianas: materiales comunes y alternativos (materia local) convirtiéndolos en generadores y transformadores de entornos activos, teniendo como base, una relectura de nuestra realidad, mucho más local y contextualizada, enlazando lo tradicional con lo contemporáneo, lo natural con lo artificial, el ser con la naturaleza (José Fernando Gómez, Natura Futura. Arquitectura, 2019). 


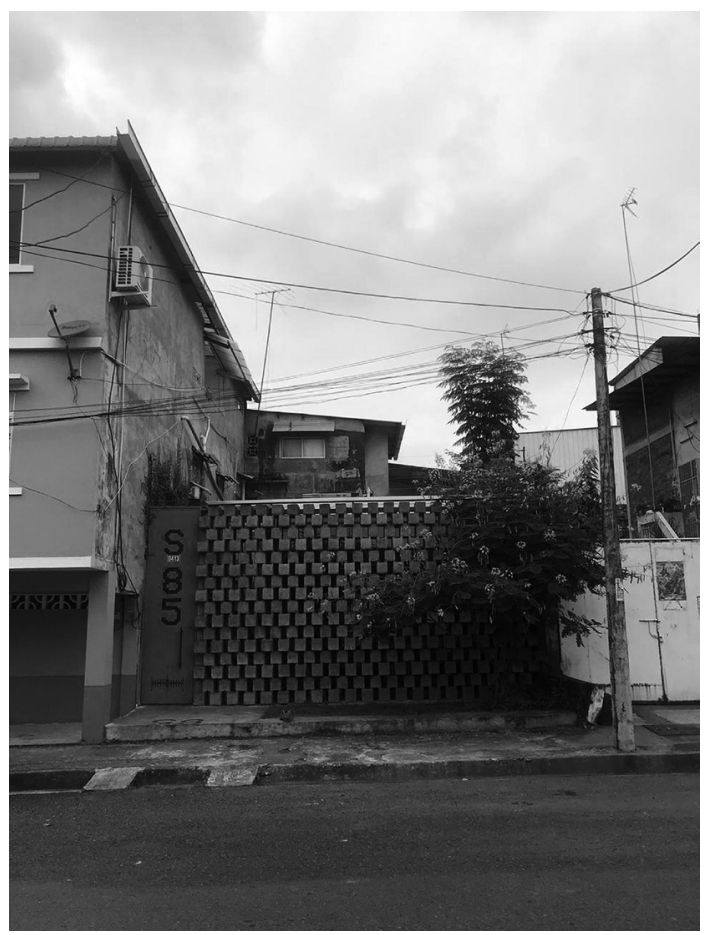

Figura 3. Casa Entre Bloques [Fotografía de Juan Carlos Bamba] (Babahoyo, Provincia de Los Ríos, Ecuador). Archivo Juan Carlos Bamba. Guayaquil, Ecuador.

Otro proyecto destacado y premiado de Natura Futura, en este caso en colaboración con el colectivo Frontera Sur Arquitectura, es La Comuna, que obtuvo el Primer Premio Hábitat Social y Desarrollo en la Bienal Panamericana de Arquitectura de Quito en el año 2018. Esta obra, ubicada en la población fronteriza de Huaquillas, en la Provincia de El Oro, al sur del Ecuador, fue realizada para una familia, donde se debía combinar su actividad de recicladores con un espacio para vivienda.

La obra en madera se configura con seis módulos iguales, a manera de contenedores, tres en planta baja para las actividades de reciclaje, y tres en planta alta para la vivienda familiar. Los arquitectos se plantearon convertir al proyecto en un hito urbano en una ciudad con profundos problemas de dotación de servicios, inseguridad y calidad de vida:

La comuna se convierte en un hito para la ciudad, debido al proceso de transformación que tuvo, con antecedentes de insalubridad y contaminación. El proyecto comunica un discurso de concientización a través de su fachada con un mensaje, generando reflexión entre lo privado y lo público a través de la arquitectura y del reciclaje (Natura Futura Arquitectura y Frontera Sur Arquitectura, 2019). 


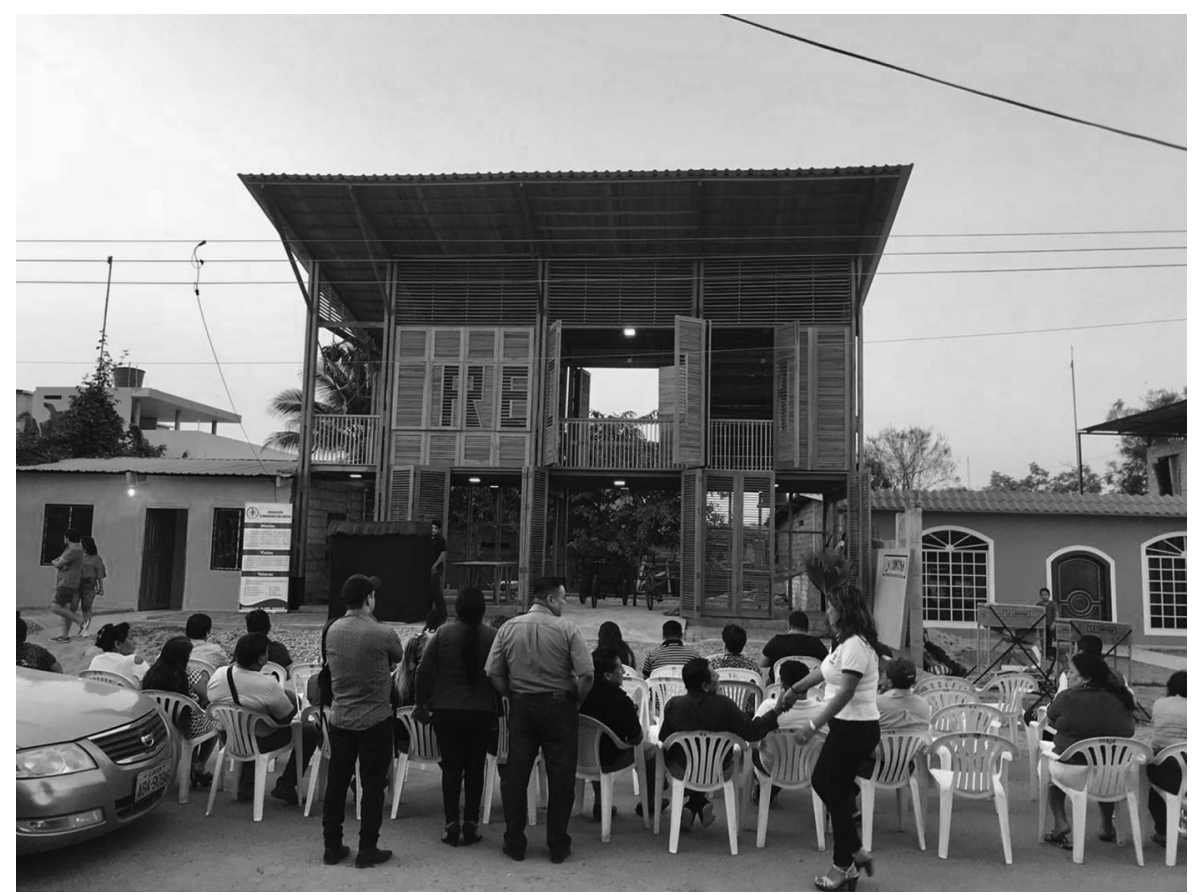

Figura 4. La Comuna [Fotografía de José Fernando Gómez] (Huaquillas, Provincia de El Oro, Ecuador). Archivo Natura Futura. Babahoyo, Ecuador.

A estos debemos sumarle proyectos como la Casa Infinita o el Asadero Rico Pollo, donde se evidencia, también, esa búsqueda de generar una arquitectura contemporánea que de sentido a una tradición de habitar, diseñar y construir.

\section{Epílogo: la originalidad en los orígenes}

En general hay un acuerdo en establecer que la Arquitectura Moderna se inicia como ruptura de la tradición y rechazo a toda referencia del pasado, a partir de la incorporación de nuevos materiales de construcción, cambios en los procesos de edificación, el cuestionamiento de las prácticas proyectuales tradicionales, la modificación de las concepciones espaciales y la relación entre la forma y la función.

Sin embargo, contraria a esta visión reduccionista, hay quienes entienden a la modernidad arquitectónica desde su actitud positiva frente al presente que se aplica a cualquier hecho 
que la refleje, más allá de su época histórica. Como indica Helio Piñón (2006), “... cuando la práctica adquiere un sentido específico que depende del uso que el autor hace de los principios teóricos y operativos -de los materiales, según el filósofo alemán Theodor Adorno- que cada época pone a su disposición" y que expresaría en principios estéticos como

... la concepción como construcción -ya no como gestión, con criterios de mímesis, de sistemas canónicos-; la abstracción como un modo de asumir la universalidad, trascendiendo pues lo particular y la forma consistente, equilibrada, en el marco de una idea de orden no reductible a la regularidad ni amparada en la jerarquía (p. 18).

En esa misma línea, Sarah Williams Goldhagen, profesora de la Universidad de Harvard, plantea la necesidad de superar el paradigma de la Arquitectura Moderna -y contemporánea- desde su reduccionismo estilístico, para pasar a entenderla como un discurso que “... afronte el fenómeno mismo de la modernidad, en lugar de rechazarlo o ignorarlo categóricamente" (Goldhagen, 2008, p. 16).

Uno de los desafíos de la arquitectura contemporánea, por tanto, es superar el proceso de ruptura de la continuidad histórica a la que fue sometida por el denominado Movimiento Moderno en el siglo XX, tal como lo han hecho estos arquitectos, quienes se plantearon (y concretaron) el desafío de desarrollar de una arquitectura capaz de adaptarse, de expresarse en un lenguaje contemporáneo y que conciliara la tradición histórica con el aporte de lo local, ya sea en lo espacial, formal o constructivo. A fin de cuentas, como decía Antonio Gaudí: "La originalidad consiste en el retorno al origen: así pues, original es aquello que vuelve a la simplicidad de las primeras soluciones".

Algo está cambiando en la arquitectura del mundo desde el sur de nuestro continente, y ese cambio se está dando en la costa del Ecuador.

\section{Notas}

1. El jurado estuvo conformado por los arquitectos Juhani Pallasmaa (Finlandia), Alberto Mozó (Chile), Mauricio Rocha (México), Florencio Compte (Ecuador) y Roberto Moscoso (Ecuador).

2. El jurado estuvo conformado por los arquitectos Alvaro Puntoni (Brasil), Luis Aldrete (México), Gloria Cabral (Paraguay), María Luisa Borja (Ecuador) e Igor Muñoz (Ecuador). 3. La Guadua angustifolia es una especie de bambú gigante utilizado tanto como material de construcción, en la estructura y cerramiento de las edificaciones, como para el proceso constructivo, en la fabricación de andamios y cimbras.

4. Es una mezcla de arcilla con paja aplicada sobre la caña abierta-denominada latillas de caña- o sobre un entretejido de ramillas de guadúa o bejuco y que se dejaba secar al sol.

5. Se refiere al bijao (Calathea lutea), planta originaria de América, semejante al plátano, de grandes hojas que por su carácter impermeable era utilizada para cubrir las techumbres de las casas. 
6. De la Provincia de Manabí, en su versión oficial o “De la puta madre”, según sus autores.

\section{Bibliografía}

Frampton, K. (2010 (4a. ed.)). Historia crítica de la arquitectura moderna. Barcelona: Gustavo Gili.

Goldhagen, S. W. (2008). Algo de qué hablar. Modernismo, discurso, estilo. Bitácora 12, 11-42. Juan, J., \& Ulloa, A. d. (1748). Relación histórica de un viaje a la América Meridional.

Natura Futura. Arquitectura. (5 de agosto de 2019). Obtenido de https://www.naturafutur arq.com/nosotros.html

Pallasmaa, J.; Mozó, A.; Rocha, M.; Compte, F. \& Moscoso, R. (2015). Acta de veredicto, categoría Diseño Arquitectónico. En Libro Ganadores BAQ 2014 (págs. 16-47). Quito: Colegio de Arquitectos del Ecuador.

Piñón, H. (2006). Teoría del Proyecto. Barcelona: Universitat Politécnica de Catalunya.

Rudofsky, B. (1964). Obtenido de http://www.homeworkmarket.com/sites/default/files/ q2/14/09/rudolfksy_awa_0.pdf

Rudofsky, B. (2000). Constructores prodigiosos. Apuntes sobre una historia natural de la arquitectura. México, D.F.: Árbol Editorial S.A. de C.V.

Viñuales, G. M. (2007). Arquitectura vernácula en Iberoamérica. Historia y persistencias. Actas del congreso internacional de Arquitectura Vernácula. "Andalucía y América, entre la tradición y la modernidad” (págs. 15-24 ). Sevilla: Ana Aranda Bernal, Francisco Ollero Lobato, Fernando Quiles García y Rafael Rodríguez - Varo Roales.

Wiener, C. (1960 [1880]). Un francés en Guayaquil. En S. G. Interamericana, El Ecuador visto por los extranjeros (Viajeros de los siglos XVIII y XIX. Puebla: J.M. Cajjica JR. S.A.

Abstract: In the 2014 Pan-American Architecture Biennial of Quito, the jury awarded the First Prize to a small house built with bamboo in a rural area of the Province of Manabí. Two years later, the DLPM Cabañón, built using brick and bamboo, was awarded. This project was integrated into a cliff facing the sea, which reconfigures the concept of space by developing a ramp that becomes a playful place to inhabit. To these we must add the work of the Natura Futura, Frontera Sur and Al Borde collectives that are raising the need to reconcile the avant-garde and the recovery of tradition, both spatial and constructive. Something is changing in the architecture of the world and that change is taking place in the south of our continent.

Keywords: tradition - avant-garde - bamboo - architectural space - contemporary architecture. 
Resumo: Na Bienal Pan-Americana de Arquitetura de Quito 2014, o júri concedeu o Primeiro Prêmio a uma pequena casa construída em bambu guadua em uma área rural da Província de Manabí. Dois anos depois foi premiado o DLPM Cabañón, feito de tijolo e bambu, integrado numa falésia voltada para o mar, que reconfigura o conceito de espaço ao desenvolver uma rampa que se torna um local lúdico para habitar. A estes, devemos somar o trabalho dos coletivos Natura Futura, Frontera Sur e Al Borde que estão destacando a necessidade de conciliar a vanguarda e o resgate da tradição, tanto espacial quanto construtiva. Algo está mudando na arquitetura do mundo e essa mudança está ocorrendo no sul do nosso continente.

Palavras chave: tradição - vanguarda - bambu guadua - espaço arquitetônico - arquitetura contemporânea.

[Las traducciones de los abstracts fueron supervisadas por el autor de cada artículo] 